\title{
The effect of unattended visual and auditory words on cross-modal naming
}

\author{
ANIMA SEN \\ Delhi University, Delhi, India 10007 \\ and \\ MICHAEL I. POSNER \\ University of Oregon, Eugene, Oregon 97403
}

\begin{abstract}
Language represents a system in which distinct sensory-specific inputs converge upon a highly overlearned common correspondence. This paper examines the influence of unattended visual and auditory words upon naming latencies. Subjects were asked to name a single auditory or visual target word that occurred at the end of a meaningful aurally presented phrase. Experiment 1 indicated redundancy gains when the same word appeared on both modalities. The redundancy gains were of about the same size for both modalities. In Experiment 1 interference was found when the unattended message was visual. Experiment 2 showed that the interference effects found in the first experiment resulted mainly from a violation of the modality-specific expectancies induced by the auditory priming phrase rather than from the unattended visual item. This result suggests that expectancies created by the auditory phrase are modality specific.
\end{abstract}

Reading and listening are among the most frequent methods by which adult humans in literate societies take in information. There are large separate literatures devoted to studies of reading and to speech perception (Kavanaugh \& Mattingly, 1972). Recent analyses of individual words presented visually and aurally suggest that both skilled listening and reading involve activation of physical, phonetic, and semantic codes (Posner, 1978). This activation has often been shown to be automatic, in the sense that it can take place without attention.

There have been studies showing that information presented to a modality other than the one to which the subject is currently responding can facilitate or retard naming the attended message. For example, when an unattended auditory digit is the same as an attended visual digit, naming performance is facilitated relative to a silent or noise-burst control (Greenwald, 1970; Mynatt, 1977). It has also been found in tasks involving words that naming latency is facilitated when an identical cross-modal word is presented simultaneously with the word to be named (Posner, Lewis, \& Conrad, 1972). Lewis (1972) also reported that related but not identical words presented simultaneously to an unattended modality could affect naming reaction times (RTs) to the attended word. In this case he found evidence of effects when the auditory information was named (visual unattended) but not such evidence for visual targets (auditory unattended).

These studies were begun while the second author was Visiting Professor of Psychology at Delhi University. The writing of this report was supported by NSF Grant BNS76-18907-A02.
The use of the auditory modality as a vehicle for the presentation of unattended information (Broadbent, 1958) rests on the idea that auditory information cannot be closed out by any obvious peripheral acts such as shutting the eyes or changing fixation. The difference suggests that unattended auditory information will have more effect on visual processing than the reverse. In addition, the high ideomotor compatibility between auditory input and speech (Greenwald, 1970) indicates that auditory input would have better access to phonetic and speech systems than visual input does. On the other hand, the evidence for visual dominance in other domains (Posner, Nissen, \& Klein, 1976) might suggest unattended visual information would affect auditory processing more than the reverse.

There is considerable evidence that semantic context affects processing of input during continuous tasks such as reading and listening. Some of this facilitation appears to be code specific. When reading phrases, subjects can easily reject homophones of contextually appropriate words as being meaningless (Baron, 1973). Homophonic words do not appear to share in facilitation produced by the context (Coltheart, in press). Semantic context, presented visually, appears to facilitate a visual or semantic rather than phonetic code. By presenting bimodal items at the end of meaningful phrases, it might be possible to obtain information on the relationship of visual and auditory input to the accumulating semantic facilitation induced by the phrase.

Our experiments deal with two issues. The first issue is the relative influence of unattended auditory and visual information on naming the attended message. 
The second is whether the semantic context obtained by listening to a meaningful passage facilitates both auditory and visual naming latencies.

\section{EXPERIMENT 1}

Subjects listened to a brief, meaningful auditory phrase. The final word of the phrase was bimodal. In the auditory condition they were to name aloud the final word, and in the visual condition they were to name the visual word that appeared simultaneously with the last word of the phrase. Naming RTs were recorded. Experiments $1 \mathrm{~A}$ and $1 \mathrm{~B}$ were exact replications except that the latter included additional emphasis upon making the naming response as quickly as possible.

\section{Method}

Stimulus materials. A list of 24 stimulus words was prepared, each of which was determined on the basis of its common semantic usage as an appropriate ending for a sentence or string of words. The list was obtained from a preliminary study in which a group of 25 Delhi University students were asked to provide the ending words of 24 incomplete sentences or phrases. The most frequently produced responses were selected as stimulus words for the main experiment. Table 1 provides the completed word strings or sentences. The last word of each of these was used as the target word.

Subjects. Twelve Delhi University students of psychology participated in Experiment $1 \mathrm{~A}$, and 12 additional students from the same source served in Experiment 1B.

Design. The basic task was to name a single visual or auditory word that followed a string of aurally presented words. Each subject served in one block of 24 trials in the visual naming task and one block of 24 trials in the auditory naming task. The order of the two tasks was counterbalanced.

Within each block, three conditions were defined. In the redundant conditions, the same word consistent with the linguistic context was presented to both the visual and auditory channels. In the neutral condition, the word consistent with context was presented to the attended channel and a word unrelated to context to the unattended channel. In the interference condition, the word related to context was presented to the unattended channel, and the subject had to name an attended word unrelated to context. These three conditions were presented equally often within each block. Unrelated words were selected from the final word of other phrases in Table 1.

Three different lists were used so that for any given subject, each of the 24 phrases occurred in only one of the three conditions (redundant, neutral, or interference). Across three subjects, each phrase occurred exactly once in each of the three conditions. The basic design was repeated for both the visual and auditory naming tasks.

Procedure. The subjects were comfortably seated on an adjustable stool, approximately $30 \mathrm{~cm}$ away from the window of a tachistoscope. The procedure involved oral presentation of 24 priming phrases to the subjects, 8 under each of the three experimental conditions. The phrase was read to the subject by the experimenter. The last auditory word following each priming string was synchronized with a 1-sec illumination of the tachistoscope that presented the visual word. The visual word was typed in capital letters.

The tachistoscope and a millisecond electronic timer (the four-digit T.K.K. Chronoscope, Model TR-4) were activated by the experimenter simultaneously with the final word. The subjects responded through a microphone connected to a voice RT apparatus (Lafayette Model 6602A) in the circuit. The
Table 1

$\begin{array}{ll}\text { Bark like a Dog } & \text { Chickens lay Eggs } \\ \text { Ring the Bell } & \text { Swim like a Fish } \\ \text { Eat with a Spoon } & \text { See with your Eyes } \\ \text { Read the Book } & \text { As a matter of Fact } \\ \text { Drink from the Glass } & \text { Tit for Tat } \\ \text { Trust in God } & \text { Open the Door } \\ \text { Laugh at a Joke } & \text { Grass is Green } \\ \text { Fly like a Bird } & \text { Food is to Eat } \\ \text { Shave with a Razor } & \text { Sew with a Needle } \\ \text { Hear with your Ear } & \text { Get milk from a Cow } \\ \text { Have a nice Time } & \text { Write with a Pen } \\ \text { The sky is Blue } & \text { Water is to Drink }\end{array}$

subjects' vocal responses through the microphone immediately stopped the time. The intertrial interval was about 10 to $15 \mathrm{sec}$. The instructions given to the subjects in Experiment $1 \mathrm{~A}$ did not put strong emphasis on speed. Experiment $1 \mathrm{~B}$ was identical to $1 \mathrm{~A}$ except that stress was placed upon responding quickly.

\section{Results}

The median RT for each subject over eight trials in each of the three conditions was calculated for both the auditory and visual naming tasks. The means of these median RTs are shown in Table 2.

An analysis of variance of the two experiments indicated no significant differences between them. In addition, there was no significant main effect of the two tasks (visual naming and auditory naming). The first result led us to combine the results of the two studies in further analysis. The latter result indicates that differences found between unattended modalities do not arise from the relative efficiency of the visual and auditory naming tasks.

The effect of condition (redundant, neutral, interference) was significant $[F(2,44)=45.8, p<.001]$. In addition, the Modality by Condition interaction was also significant $[F(2,44)=6.24, p<.01]$. Tukey tests were used to explore the relationship between the three conditions for each task. It was found that the redundant condition was significantly faster $(p<.01)$ than the neutral condition for both tasks. However, the interference condition was only slower than the neutral condition in the auditory naming task $(p<.01)$. Although the interference condition for visual naming shows a rather long mean RT in Table 1 , it does not differ significantly from the neutral condition by Tukey

Table 2

Mean of the Median Subject Reaction Times for the Various Conditions of Experiments 1A and 1B

\begin{tabular}{lrrrrrrr}
\hline & \multicolumn{3}{c}{ Auditory Task } & & \multicolumn{3}{c}{ Visual Task } \\
\cline { 2 - 3 } \cline { 6 - 8 } & \multicolumn{1}{c}{$\mathrm{R}$} & \multicolumn{1}{c}{$\mathrm{N}$} & \multicolumn{1}{c}{$\mathrm{R}$} & \multicolumn{1}{c}{$\mathrm{N}$} & $\mathrm{I}$ \\
\hline Experiment 1A & 474 & 501 & 573 & & 507 & 550 & 548 \\
Total Errors & 0 & 0 & 2 & & 0 & 0 & 6 \\
Experiment 1B & 414 & 430 & 546 & & 453 & 483 & 531 \\
Total Errors & 0 & 0 & 2 & 0 & 0 & 2 \\
\hline
\end{tabular}

Note $-R=$ redundant,$N=$ neutral, $I=$ interference . 
test. It appears clear that the interaction between task and condition results from the interference effect being found only during the auditory naming task.

The total errors in both experiments were too few to reveal anything of interest. The instruction to "go fast" did not produce the expected increase in errors. Obviously, the naming task is a highly compatible one and the low error rates reflect that fact.

\section{Discussion}

The most important and striking effect of this experiment is evidence that an unattended redundant stimulus has effects upon processing the attended message. The effect of an unattended auditory item has been demonstrated many times in the dichotic listening literature. However, the automatic effects of unattended visual items while listening has not been very thoroughly documented. Lewis (1972) has the only report of this, and his study involved the use of unrelated words. It should be remembered that subjects in the auditory shadowing condition of the present study were never required to read any words. They were instructed merely to keep their eyes open. While most subjects were aware that visual information was present during the study, at least one subject was surprised when told after the experiment that visual words had been presented during the task.

Interference effects from unattended visual input are clearly much larger than from unattended auditory input. These findings might suggest that unattended visual input has stronger effects on auditory processing than the reverse. However, before this can be concluded, a confound in the neutral conditions of the two experiments must be examined.

\section{EXPERIMENT 2}

The interference conditions of Experiment 1 differ from the neutral condition in two ways: first, because the attended information is unrelated to the context, and second, because the unattended information is related to the context. The differences found between the interference conditions in the visual and auditory task could be because an unattended visual word in context is processed more readily or because it is more detrimental to shift semantic context when the target word is auditory than when it is visual. Since the semantic priming phrase is always auditory, it could be that the expectancy developed by the phrase is specific to the auditory modality, making a semantic switch very detrimental when it occurs in the auditory-attend condition.

To separate these ideas, we introduced a new control condition in which the final word in both the attended and unattended modalities was unrelated to the semantic context of the priming phrase. The new control condition was compared with the neutral and interference conditions used previously. If the effects found in Experiment 1 were due to the violation of semantic context on the attended modality, the control condition should be similar to the interference conditions of Experiment 1. If the effects were due to greater influence from the unattended word when it fits the semantic context, the control should resemble the previous neutral condition.
Table 3

Mean of the Median Reaction Times for the Various Conditions of Experiment 2

\begin{tabular}{cccccccc}
\hline \multicolumn{3}{c}{ Auditory Task } & & \multicolumn{4}{c}{ Visual Task } \\
\cline { 7 - 8 } \cline { 5 - 7 } $\mathrm{N}$ & $\mathrm{C}$ & $\mathrm{I}$ & & $\mathrm{N}$ & $\mathrm{C}$ & $\mathrm{I}$ \\
\hline 505 & 573 & 596 & & 603 & 612 & 620 \\
\hline
\end{tabular}

Note $-N=$ neutral, $C=$ control, $I=$ interference.

\section{Method}

Subjects. A new group of 12 Delhi University students participated in this experiment as subjects.

Design. The experiment was designed and run exactly as before, except that the redundant condition was replaced by the new control condition. In the control condition, the final words in both modalities were items selected from those used in Table 1, but they were unrelated to the phrase.

\section{Results}

The mean of the median RTs for subjects in both tasks and the three conditions are shown in Table 3.

An ANOVA of these scores revealed significant effects of modality $[F(1,11)=62, p<.001]$, condition $[\mathrm{F}(2,11)=21.5, \mathrm{p}<.001]$, and the interaction of modality and condition $[F(2,11)=10.6, p<.001]$.

Unfortunately, the subjects were faster with auditory targets in this experiment. The main differences between the two naming tasks was that for the auditory-attend task, the control and interference conditions were significantly longer than the neutral condition $(\mathrm{p}<.01$, by Tukey test), but they did not differ significantly from each other. In the case of the visual-attend task, there was no significant difference between conditions.

Error rates in this experiment were too small for analysis. Only two errors were made in the auditory naming condition and six in the visual naming condition.

\section{Discussion}

The results of Experiment 2 indicate that the interference effects found for the auditory naming task in Experiment 2 were due to a violation of semantic context on the attended message. The fact that this occurs only when the auditory word is to be named indicates that the expectancy set up by the context is modality specific. If the modality is violated, one finds no advantage of semantic context, even though the switch of modality occurs for every trial in the block. This is remarkable evidence for the specificity of the semantic context provided by the phrase. It should be noted that there is a tendency for the interference condition in the auditory naming condition to be longer than the control and neutral conditions, although this trend is not significant. Since auditory RTs are very fast in this study, the effectiveness of the unattended visual message may be reduced.

\section{CONCLUSIONS}

Two questions were raised in the introduction. First, does information in an unattended modality influence processing of attended information while processing meaningful phrases? The answer to this question is provided by the redundancy gain found in Experiment 1. Both unattended visual and auditory information improve RTs to the relevant modality. Although the effect of unattended visual information is slightly larger, there is no significant difference between the two modalities. 
These results correspond with those found by Posner et al. (1972) in their task of processing random words. There seems to be no doubt now that unattended visual information is processed, even when the task is confined to listening and there is no incentive given to process this information. This provides additional confirmation of the automatic nature of reading in the skilled person. However, it should be recognized that this redundancy gain effect takes place in a situation where both the linguistic context and the attended modality serves to prime the unattended message.

The evidence for automatic processing would be stronger if unattended messages that are not identical to the attended message produced effects due to their semantic relationships to the priming string. This result was reported by Lewis (1972). The interference conditions were designed to assess this possibility. However, the results of Experiment 2 show that interference effects found in Experiment 1 were not due to the unattended message but to violation of the expected word in the attended message. Although in the case of an unattended visual word, RT to the interference condition of Experiment 2 was somewhat longer than to the control condition, this effect was not significant.

The results of Experiment 2 indicate that semantic facilitation in our experiments is modality specific. In the auditoryattend condition, presenting a word unrelated to context dramatically increased RT. However, if the subject is required to name a visual word, whether it fits the context does not matter. It is well known that semantic facilitation can occur to cross-modal items in other paradigms and that the degree of modality specificity of context tends to be reduced with delay (Kirsner \& Smith, 1974). On the other hand, our evidence for modality specificity agrees with recent studies of reading that argue that semantic context operates on physical, not phonetic, codes (Baron, 1963; Coltheart, in press). It may well be that during the absorption of information from reading or listening, context begins by facilitating only those items sharing the same physical code. As the semantic information is abstracted, one finds facilitation of all items sharing the same meaning. The technique of bimodal presentation used here may aid in developing the time course of these effects during both reading and listening. Bimodal presentation has the advantage over the use of homophones, for example, of providing words with distinctly different physical codes that have common phonetic and semantic interpretations.

\section{REFERENCES}

Baron, J. Phonemic stage not necessary for reading. Quarterly Journal of Experimental Psychology, 1973, 25, 241-246.

Broadbent, D. E. Perception and communication. London: Pergamon Press, 1958.

Colthe ART, M. Lexical access in simple reading tasks. In G. Underwood (Ed.), Strategies on information processing. New York: Academic Press, in press.

Greenwald, A. G. A double stimulation test of ideomotor theory with implications for selective attention. Journal of Experimental Psychology, 1970, 84, 392-398.

Kavanaugh, J. F. \& Mattingly, I. G. Language by ear and by eye. Cambridge, Mass: MIT Press, 1972.

Kirsner, K., \& SMith, M.C. Modality effects in word recognition. Memory \& Cognition, 1974, 2, 637-640.

LEWIS, J. L. Semantic processing with bisensory stimulation. Journal of Experimental Psychology, 1972, 94, 455-457.

MynatT, B. T. Reaction times in a bisensory task: Implications for attention and speech perception. Journal of Experimental Psychology: Human Perception and Performance, 1977, 3, 316-324.

Posne r, M I. Chronometric exploration of mind. Hillsdale, NJ: Erlbaum, 1978.

Posner, M.I., Lewis, J. L., \& Conrad, C. Component processes in reading: A performance analysis. In J. F. Kavanaugh \& I. G. Mattingly (Eds.), Language by ear and by eye. Cambridge, Mass: MIT Press, 1972.

Posner, M. I., Nissen, M. J., \& KLein, R. Visual dominance: An information processing account of its origins and significance. Psychological Review, 1976, 83, 157-171.

(Received for publication March 26, 1979.) 